In der Staatsanwaltschaft Saarbrücken habe ich bereits im Sonderdezernat Häusliche Gewalt ein Praktikum absolviert. Seitdem beschäftigte ich mich verstärkt mit Themen der Gleichberechtigung und des Opferschutzes bei Gewaltstraftaten.

Am djb schätze ich insbesondere, dass junge Studentinnen und Berufseinsteigerinnen von Juristinnen mit Berufserfahrung Unterstützung und Perspektiven erhalten. Um diesen Prozess aktiv zu unterstützen, möchte ich als Beisitzerin im Bundesvorstand kandidieren.

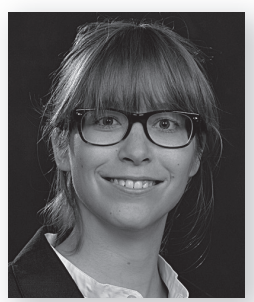

Denise Cordes, Hauptamtliche Leiterin von Justitia Mentoring, dem Frauenförderprogramm an der Rechtswissenschaftlichen Fakultät der AlbertLudwigs-Universität Freiburg i.Br. und Autorin im sechsköpfigen Team einer täglichen juristischen Presseschau bei LegalTribuneOnline.

Geb. 1983 in Bochum, Studium der Rechtswissenschaften mit europarechtlichem Wahlfach an der Ruhr-Universität Bochum, Hilfskraft am Lehrstuhl für Europa- und Völkerrecht (Prof. Dr. Adelheid Puttler, LL.M.). Teilnahme (2006) und anschließende Leitung (2007/ 2008) des Bochumer Philip C. Jessup International Law Moot Court CompetitionTeams. Erstes Staatsexamen 2008. Bis 2012 wissenschaftliche
Mitarbeiterin am Institut für Staatswissenschaft und Rechtsphilosophie, Universität Freiburg; zuvor am Lehrstuhl für Öffentliches Recht, Rechtssoziologie und Rechtsphilosophie an der Universität Bochum (jeweils Prof. Dr. Ralf Poscher). Dissertationsprojekt im Verfassungs- und Völkerrecht zum strafrechtlichen Verbot der Völkermordleugnung. Die weiteren Interessenschwerpunkte liegen im allgemeinen Staatsund Verwaltungsrecht, der feministischen Rechtswissenschaft und der Rechtsphilosophie (Strafrecht).

In Freiburg im Bereich der universitären Selbstverwaltung als Mitglied der Studienkommission und des Fakultätsrates der Rechtswissenschaftlichen Fakultät seit 2010 bzw. 2012 tätig. djb-Mitglied seit 2013. Ehrenamtliches Mitglied im Leitungsteam von Justitia Mentoring und Mentorin im Programm seit 2010 .

Ein besonderes Anliegen wäre mir bei den jungen Juristinnen eine verstärkte Zusammenarbeit mit den Rechtswissenschaftlichen Fakultäten. Mittels der Einführungs- und Orientierungsveranstaltungen kann der djb bei Studentinnen stärker hervortreten. Gerade jungen Frauen stehen dem Konzept der „Frauenförderung“ oft skeptisch gegenüber; gleichzeitig ist der Kontakt mit weiblichen Vorbildern in diesem Rahmen - insbesondere auch mit Blick auf die Vorstellung unterschiedlichster Berufsbilder - und das Netzwerken unter Frauen eine wichtige Erfahrung.

\title{
Sitzung des Regionalgruppenbeirates vom 26. bis 28. April 2013 in Augsburg
}

\section{Daniela Leukert-Fischer \\ Rechtsanwältin, Vorstand Regionalgruppenbeirat, Vorstand RG Karlsruhe, Bensheim}

Der Regionalgruppenbeirat (RGB) des djb tagt einmal jährlich mit dem Ziel des Informations- und Erfahrungsaustausches zwischen den Regionalgruppen und Landesverbänden. In diesem Jahr kamen vom 26. bis 28. April insgesamt 22 Delegierte aus ganz Deutschland in Augsburg zusammen.

Nachdem das Treffen am Freitagabend mit einem ersten informellen Abendessen begonnen hatte, kamen die Teilnehmerinnen am Samstagmorgen im Ratskeller des Augsburger Rathauses zu ihrer Sitzung zusammen.

Die Präsidentin des djb, Ramona Pisal, berichtete über die Arbeit des djb auf Bundesebene und insbesondere über ihre Tätigkeit im letzten Jahr und die Besuche der verschiedensten Veranstaltungen wie auch die Teilnahme an diversen Hauptversammlungen im Rahmen unseres Projekts „Aktionärinnen fordern Gleichberechtigung “. Auch unsere Vizepräsidentin, Eva Schübel, war zur Sitzung angereist, um die Teilnehmerinnen zu begrüßen und einen Zwischenstand zur erfolgreichen
Aktion „Rote Roben“ sowie zum Netzwerk der Gleichstellungsbeauftragten zu geben.

Bundesgeschäftsführerin Anke Gimbal gab einen Überblick über die geplanten Veranstaltungen, insbesondere den nächsten Bundeskongress in Leipzig vom 26. bis 29. September

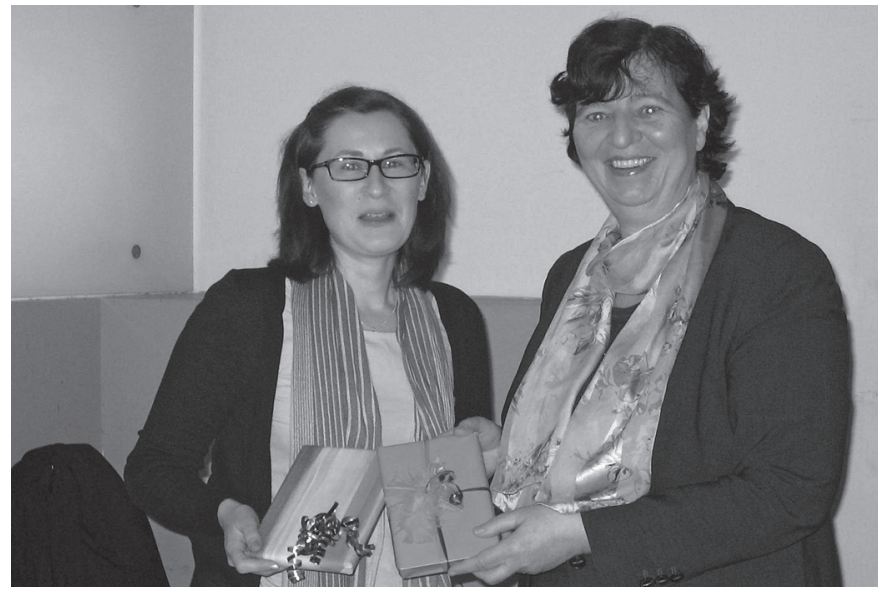

A Verabschiedung von Rechtsanwältin und vereidigte Buchprüferin Birgit Kersten (rechts), Brake, nach sechs Jahren Amtszeit als RGB-Vorstandsmitglied, mit Rechtsanwältin Daniela Leukert-Fischer, Bensheim. Bild: Christina Lorenz. 
2013. Sie erinnerte darüber hinaus noch einmal an die im Rahmen des Kongresses in Potsdam eröffnete Wanderausstellung "Juristinnen in der DDR“, die von den Regionalgruppen und Landesverbänden präsentiert werden kann und sehr erfolgreich angenommen wird.

Im Anschluss an die Berichte der angereisten Bundesvorstandsmitglieder hielt Sabine Overkämping, Mitglied der Kommission Öffentliches Recht, Europa- und Völkerrecht und Vorsitzende der Fachgruppe Europarecht, einen hochinteressanten Vortrag zur Struktur und Arbeitsweise der Kommission. Sie stellte die Wahlprüfsteine der Kommission vor, die in der djbZ veröffentlicht werden. Positiv war der anschließende rege Austausch zwischen den Teilnehmerinnen und dem Kommissionsmitglied. Nicht zuletzt wurde auch über die kurzen Stellungnahmefristen diskutiert, die die Arbeit der Kommissionen erschweren, da in immer kürzer werdenden Fristen qualifizierte und rechtlich komplexe Stellungnahmen gefertigt werden müssen. Die Frage, ob man nicht eine hauptamtliche Hilfskraft den Kommissionen zur Seite stellen solle, wurde in diesem Zusammenhang aus dem Teilnehmerkreis (nicht abschließend) diskutiert.

Sabine Overkämping forderte zudem die Regionalgruppen und Landesverbände auf, Mitglieder der Kommission in die Region einzuladen. Sie berichten gerne vor Ort über ihre Arbeit. Die Mitglieder der Kommissionen können über die Geschäftsstelle erfragt und kontaktiert werden.

Mittags wurden die Teilnehmerinnen im Fürstenzimmer des Rathauses der Stadt Augsburg von Bürgermeister Peter Grab empfangen. Im Anschluss an seine Begrüßung übernahm die Präsidentin Ramona Pisal das Wort. In einer persönlichen, sehr humorvollen sowie pointierten Ansprache stellte sie neben der Arbeit des djb auch die Stellung der Frauen in der Gesellschaft und insbesondere in der Biographie des Jakob Fugger heraus, der eine Frau brauchte, um seine Ziele zu erreichen. Erst die Heirat mit Sibylle Arzt im Jahre 1498 ebnete ihm den Weg zum Großbürgertum Augsburgs und ermöglichte ihm den lang ersehnten Sitz im Stadtrat (Näheres auch mit Fotos vom Empfang unter www.augsburg.de). Die Teilnehmerinnen waren sich einig, dass ihre Worte: „Vergessen Sie die Frauen nicht! " wohl noch nachhallen werden.

Der Samstagnachmittag war ganz dem Erfahrungs- und Ideenaustausch zwischen den Regionalgruppen und Landesverbänden vorbehalten. Die Vertreterinnen der Regionalgruppen und Landesverbände erzählten jeweils ausführlich von ihrer Arbeit im vergangenen Jahr, die durchgeführten Veranstaltungen sowie die Entwicklung der Mitgliederzahlen und Mitgliederbeteiligung. Im Fokus standen dabei die Gründung einer neuen Regionalgruppe in Madrid, der Kontakt zu den Mitgliedern in der Regionalgruppe und die Zusammenarbeit mit anderen Verbänden z.B. bei einzelnen Veranstaltungen.

Neben dem langen Sitzungstag und einer kleinen Führung durch das Rathaus am Mittag bot sich dann am späten Samstagnachmittag die Möglichkeit, die Stadt Augsburg durch einen hochinteressanten Stadtrundgang insbesondere durch

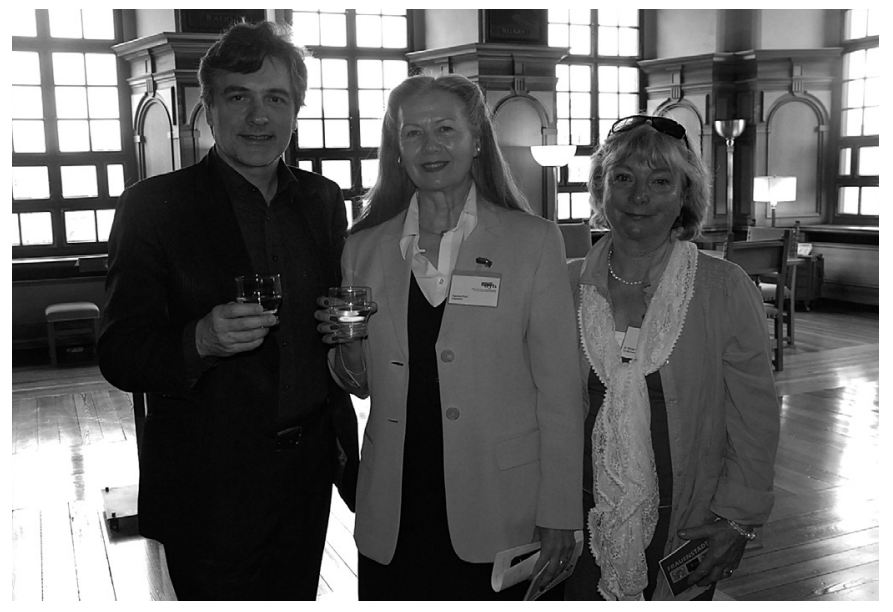

- Empfang im Augsburger Rathaus: Bürgermeister Peter Grab, Präsidentin Ramona Pisal und RG-Vorsitzende Dr. Monika Zumstein (von links). Bild: Stadt Augsburg.

die Besichtigung der Fuggerei, der ältesten bestehenden Sozialsiedlung der Welt, besser kennen zu lernen.

Der nächste Sitzungstag, der Sonntag, begann mit dem Bericht des Vorstands des Regionalgruppenbeirates. Er berichtete über seine Tätigkeit im vergangenen Jahr wie zum Beispiel die Information der Regionalgruppen und Landesverbände durch das letzte RGB-Rundschreiben und den regelmäßigen Besuch der Bundesvorstandssitzungen. Die Themen Mitgliederwerbung und „Strategie 2020“ (Arbeitstitel) waren auch innerhalb des Bundesvorstands behandelt worden, so dass die Teilnehmerinnen auf den aktuellen Stand der Diskussion innerhalb des Vereins gebracht wurden. Die Umfrage zur und in der djbZ war hier ein Ergebnis der Beratungen und man erinnerte die Teilnehmerinnen, sich an der Umfrage zu beteiligen, gerne auch anonym, um ein allumfassendes Meinungsbild zu erhalten.

Jedoch nicht nur die Ausrichtung des Vereins wurde an diesen Sitzungstagen behandelt, sondern auch organisatorische Fragen.

Im Hinblick auf die Verwaltung des Vereins wurde von den Teilnehmerinnen einhellig angeregt und bereits dem Bundesvorstand mitgeteilt, über einen Wechsel der Hausbank, momentan Deutsche Bank AG, nachzudenken.

Bei einigen Regionalgruppen oder Landesverbänden wiesen wir erneut auf die Wichtigkeit der Aktualität der Homepages hin. Vergangene Veranstaltungstermine sollten aus der Rubrik „Aktuelle Termine“ gelöscht werden. Meist gerät die Homepage bei der täglichen Vorstandsarbeit in Vergessenheit oder die Kapazitäten sind einfach nicht vorhanden. Sofern dagegen lediglich technische Probleme die Ursache sind, können diese an den Vorstand des Regionalgruppenbeirates oder die Geschäftsstelle herangetragen werden.

In diesem Jahr standen zudem die Vorstandswahlen an. Im zweijährigen Turnus werden die drei Mitglieder des Vorstands des Regionalgruppenbeirats in der Sitzung gewählt. $\mathrm{Zu}$ Beginn der Wahl wurde Birgit Kersten vielmals für die hervorragende Vorstandsarbeit in den vergangenen 6 Jahren gedankt. Ihre Amtszeit lief aus. Die ehemaligen Vorstands- 
mitglieder Andrea Kirberger (RG Dresden) und Daniela Leukert-Fischer (RG Karlsruhe) kandidierten erneut und wurden im Amt bestätigt. Neu in den Vorstand wurde Dr. Annette Matthias-Werner (RG Brüssel) gewählt, die bereits ankündigte, die Europafragen auch im RGB mehr in den Mittelpunkt rücken zu wollen.

Nach wie vor auf großes Interesse stößt die Aktion „Bundesvorstand intern“, bei der einer Regionalgruppenvertreterinnen die Möglichkeit geboten wird, an Bundesvorstandssitzungen teilzunehmen. Für die nächsten Bundesvorstandssitzungen sind die Regionalgruppen Köln, Bodensee, Bonn und Brandenburg (in dieser Reihenfolge) ausgelost worden. Auch für die Wahl des nächsten Sitzungsortes gab es zahlreiche Bewerbungen. Schlussendlich konnte sich Osnabrück als Austragungsort 2014 mehrheitlich durchsetzen.
Insgesamt war die Sitzung des RGB in Augsburg wie schon in den letzten Jahren eine Bereicherung und hat den Kontakt zwischen den einzelnen Regionalgruppen und Landesverbänden sowie zum Bundesvorstand und den Kommissionen abermals vertieft. Es ist hervorzuheben, dass die Institution RGB in vollem Umfange die Funktionen erfüllt, die sich die Mitglieder bei der damaligen Gründung davon versprochen hatten.

Besonderer Dank für die Vorbereitung der großartigen Veranstaltung gilt Dr. Monika Zumstein (RG München/Südbayern), die sich in besonderem Maße dafür eingesetzt hat, dass sowohl der Sitzungsort, das Hotel wie auch der gesamte Aufenthalt in Augsburg allen Teilnehmerinnen lange in positiver Erinnerung bleiben werden.

\section{Regionalgruppenbeirat - Vorstand -}

\section{Dr. Annette Matthias MCL}

geb. 24. Februar 1950 in Gütersloh/Westfalen, verheiratet, drei Kinder.

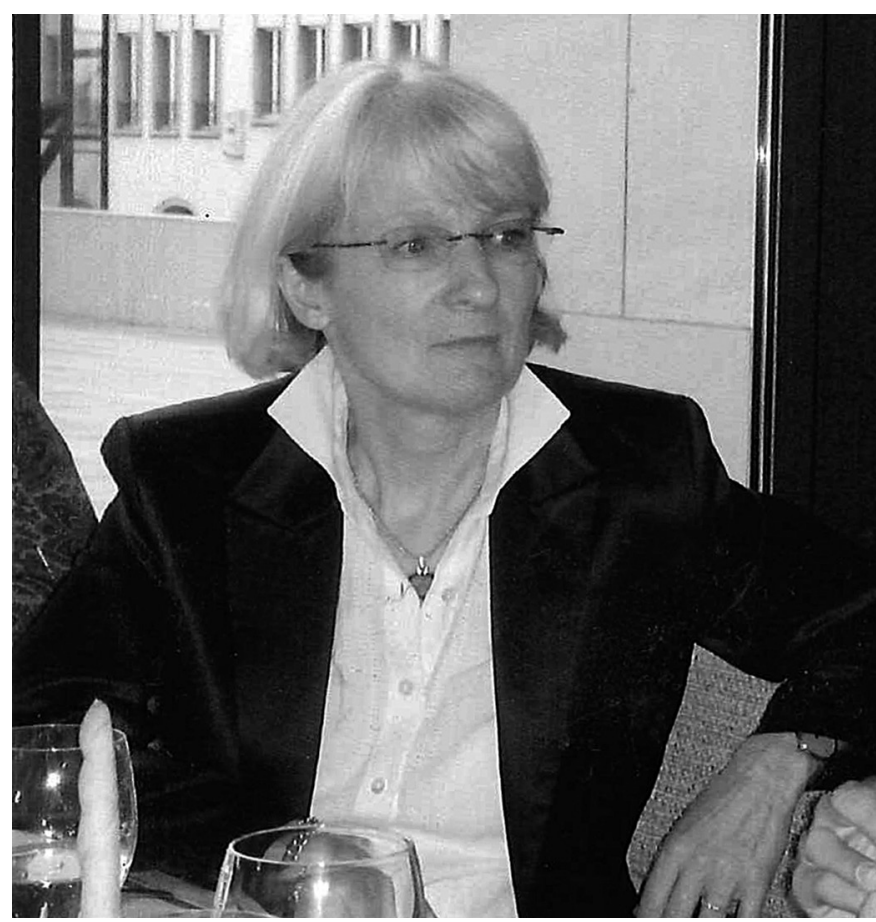

- Kindheit und Schulzeit in Mainz.

- 1968 Abitur am altsprachlichen Rhabanus-Maurus-Gymnasium in Mainz

- 1970-1974 Studium der Rechtswissenschaften in Mainz, Freiburg, Lausanne.

- 1974 Erstes Staatsexamen in Freiburg.

n 1974-1977 Promotion zu einem europarechtlichen Thema.

- 1975-1976 Studium am Europakolleg in Brügge.

- 1977-1979 Referendarausbildung in Berlin, anschließend Rechtsanwaltstätigkeit in Berlin.

- 1980-1981 Studium an der George Washington Law School in Washington DC.

- 1981-1984 Tätigkeit in verschiedenen Anwaltsbüros in Köln und Düsseldorf.

- Heiratsbedingter Umzug nach Kuwait, dort Mitarbeit in kuwaitisch-palästinensischer Anwaltskanzlei.

- 1986-1988 Unterbrechung der Berufstätigkeit.

- 1988-2012 Beamtin bei der Europäischen Kommission in Brüssel.

- Seit Januar 2012 Hauptverwaltungsrätin der Europäischen Kommission i.R., Mitarbeit in energierechtlicher Kanzlei in Brüssel.

- Sprachen: Deutsch, Französisch, Englisch, Schwedisch fließend. Grundkenntnisse in Spanisch und Portugiesisch. 